International Journal of Modern Physics A

(C) World Scientific Publishing Company

\title{
ANALYTIC RESULTS FOR THE EFFECTIVE ACTION
}

\author{
STEVEN K. BLAU* and MATT VISSER ${ }^{\dagger}$ \\ Theoretical Division, Los Alamos National Laboratory, Los Alamos, New Mexico 87545 \\ ANDREAS WIPF $\ddagger$ \\ Max Planck Institut für Physik und Astrophysik, Werner Heisenberg Institut für Physik, \\ D-8000 München 40, Germany
}

Received 12 July 1990

\begin{abstract}
Motivated by the seminal work of Schwinger, we obtain explicit closed form expressions for the one-loop effective action in a constant electromagnetic field. We discuss both massive and massless charged scalars and spinors in two, three, and four dimensions. Both strong field and weak field limits are calculable. The latter limit results in an asymptotic expansion whose first term reproduces the Euler-Heisenberg effective Lagrangian. We use the zeta function renormalization prescription, and indicate its relationship to Schwinger's renormalized effective action.
\end{abstract}

Published version: IJMPA 6 (1991) 5409-5433.

doi:10.1142/S0217751X91002549

12 July 1990; arXiv-ed 16 July 2009; LATEX-ed October 25, 2018

\section{Introduction}

In the path integral formulation of fermionic field theories, one is forced to confront the determinant of the Dirac operator, while for spin- 0 bosons one encounters the determinant of the gauged Laplacian. In the absence of gauge fields, and if the spacetime geometry is not an issue, this determinant is an irrelevant constant. However, there is still a good deal of work to be done toward elucidating the dependence of these determinants on background gauge fields and gravitational fields. These determinants are related to the one-loop effective action via $S_{\text {eff }} \propto \ln \operatorname{det} D$, and, in the

*Present address [2009]: AIP, Washington DC; e-mail: sblau@aip.org.

${ }^{\dagger}$ Present address [2009]: School of Mathematics, Statistics, and Operations Research,

Victoria University of Wellington, New Zealand; e-mail: matt.visser@msor.vuw.ac.nz; url: http://homepages.msor.vuw.ac.nz/ visser/

¥Present address [2009]: Theoretisch-Physikalisches-Institut, Friedrich-Schiller-Universität Jena, e-mail: 07743 Jena, Germany, e-mail: wipf@tpi.uni-jena.de; url: http://www2.uni-jena.de/ p5anwi/ 
guise of the one-loop effective action, have been the subject of considerable efforts dating back at least to the seminal works of Euler and Heisenberg, 11 Weisskopf, 2 and Schwinger. 3

Only in two dimensions is the situation reasonably well understood. The particularly simple geometry of compact two-dimensional Riemann surfaces makes it possible to express the gauge-field-free Dirac determinant in terms of Riemann

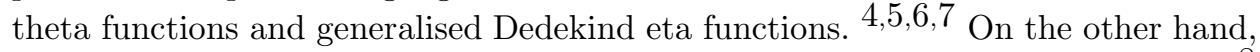
in the plane the determinant for localized gauge fields is given by Schwinger's 8 famous result $S_{\text {eff }} \propto \int A^{\mu} A_{\mu}$. Combining the effects of non-trivial topologies and non-zero field strength has been discussed in reference 9 .

In higher dimensions, only particular background fields can be handled. For example, the effect of conformal metric deformations on the effective action is discussed in references 10 and 11 . Schwinger ${ }^{3}$ has considered the effective action for constant electromagnetic field strength and for a plane wave of electromagnetic radiation, both in flat four-dimensional Minkowski space. Four-dimensional instanton solutions have also been considered. 1213 14

In this paper we shall be interested in obtaining explicit analytic results. Accordingly, we are forced to restrict our attention to particular backgrounds: flat spaces with zero-field or constant field. We use the zeta-function regularisation of determinants of second-order elliptic operators. The Dirac operator, $\not D$, is first order, but we shall define $\operatorname{det} \not D \equiv \sqrt{\operatorname{det} \not D^{2}}$. In order that $\not D^{2}$ may be an ellicptic operator, we shall work in Wick rotated Euclidean spacetime. In section 2 we consider (non-)Abelian gauge fields defined on multidimensional tori $\left(S^{1}\right)^{d}$. The non-trivial topology of these tori allows for the possibility of harmonic gauge potentials, that is, potentials which have vanishing field strength but which are not pure gauge. The existence of these harmonic gauge potentials is associated with the possibility of encountering non-trivial Wilson loops. On multidimensional tori, such gauge potentials are constant (up to a gauge transformation), thus allowing explicit construction of the eigenspectrum, zeta function, and effective action. The dependence of the effective action on these nontrivial Wilson loops may be viewed as a generalized Aharonov-Bohm effect. In sections 3 through 7, we consider gauge fields with constant field strength in arbitrarily many dimensions, working our way up from two dimensions. In all these cases we shall determine the eigenspectra of the gauged Laplacian and Dirac operator and shall calculate the associated zeta functions explicitly. Using special properties of zeta functions may often give the determinant and effective Lagrangian density in closed form.

We shall also discuss the "folk theorem" $\mathcal{L}_{\text {eff }} \sim B^{d / 2} \ln B$, and will point out a number of situations in which it is violated. The physical implications of the logarithmic term are discussed in reference 15 . We shall show that this folk theorem is generally true in even numbers of dimensions, though there are exceptions, such as the scalar particle in two dimensions. In odd numbers of dimensions however, the logarithmic term is absent, and generically we obtain $\mathcal{L}_{\text {eff }} \sim B^{d / 2}$. 


\section{Field-Free Gauge Potentials}

In this section we shall see that a generalized Aharonov-Bohm effect can influence the one-loop effective action of a system, even though the electric field strength is everywhere zero. Notice that an Abelian gauge potential is gauge-equivalent to the sum of its harmonic, monopole and coexact pieces

$$
A=H+A^{M}+\delta \chi
$$

The number of independent harmonics equals the first Betti number and is thus intimately related to the topology of the spacetime manifold. We shall be interested in gauge potentials on $d$-dimensional tori, in which case there are $d$ independent harmonics which may be chosen to be constant one-forms. In this section we shall further specialise to the zero-field case so that the monopole contribution and $\chi$ are both zero.

We begin with (constant) harmonic potentials defined on the $d$-dimensional torus $R^{d} / \Lambda$, where the lattice $\Lambda$ consists of points of the form $L \sum n^{j} \vec{E}_{j}$, the $n^{j}$ being integers and the $\vec{E}_{j}$ being $d$ linearly independent vectors in $R^{d}$. It is, of course, possible to set $L=1$ but we prefer to keep this prefactor in order to track the effect of scaling the lattice. Note that a constant field-free potential is not pure gauge since the "would be" gauge transformation is not single valued. These potentials are closed but not exact. Equivalently one sees that the Wilson loop $W\left(\gamma_{j}\right)=\exp \left(-i \oint_{\gamma_{j}} \vec{A} \cdot d \vec{x}\right)=\exp \left(-i \vec{A} \cdot \vec{E}_{j} L\right)$ evaluated on the closed noncontractible loop $\gamma_{j}$ from $\overrightarrow{0}$ to $L \vec{E}_{j}$ is gauge invariant, and is therefore an obstruction to gauging the potential away to zero.

The eigenvalues of $-D^{2}$ and $\not D^{2}$ are in fact identical for zero field. On the multidimensional torus with periodic boundary conditions, $\psi(\vec{x})=\psi(\vec{x}+\vec{\mu}), \vec{\mu} \in \Lambda$, the eigenvalues are easily computed to be

$$
\lambda_{n}=\left(\frac{2 \pi}{L}\right)^{2} \cdot g^{i j}\left(n_{i}-a_{i}\right)\left(n_{j}-a_{j}\right) ; \quad \vec{n} \in Z^{d},
$$

where the matrix $\left(g^{i j}\right)$ is the inverse of $g_{i j}=\vec{E}_{i} \cdot \vec{E}_{j}$; and $a_{i}=\frac{L}{2 \pi} \vec{E}_{i} \cdot \vec{A}$. To compute the corresponding effective action one defines

$$
S_{\mathrm{eff}}=\frac{1}{2} \ln \operatorname{det}\left(-D^{2}\right)=-\left.\frac{1}{2} \frac{d}{d s} \zeta(s)\right|_{s=0,}
$$

where $\zeta(s)$ is the zeta function associated with $-D^{2}$,

$$
\zeta(s)=\operatorname{tr}^{\prime}\left(-\frac{D^{2}}{\mu^{2}}\right)^{-s}=\sum^{\prime}\left(\frac{\lambda_{n}}{\mu^{2}}\right)^{-s}
$$

It should emphasised that the zeta function definition embodied in (4) implies both a regularization and a renormalization. When comparing the zeta function result with effective actions calculated using other renormalization prescriptions (e.g., Schwinger's) one should always bear in mind that different renormalization 
prescriptions will yield effective actions that may differ by a finite renormalization. In particular, the quantity $\mu$ appearing in (4) is a renormalization scale, which has been introduced to keep the zeta function dimensionless, and the dependence of $S_{\text {eff }}$ on $\mu$ corresponds to a finite renormalization. The dependence on the renormalization scale is logarithmic and proportional to the conformal anomaly (see e.g., reference $\frac{16}{}$ ). In order that dimensionful quantities may be properly displayed throughout, we shall retain this normalization scale in all our calculations.

In the present case the zeta function associated with the differential operator $-D^{2}$ is given in terms of a relatively well understood special function by

$$
\zeta(s)=2\left(\frac{\mu L}{2 \pi}\right)^{2 s} \zeta_{E}(s, \vec{a}) .
$$

The prefactor 2 arises from summing over both particle and antiparticle states, while the symbol $\zeta_{E}(s, \vec{a})$ denotes the generalized Epstein zeta function, $17 / 18$ defined by the sum

$$
\zeta_{E}(s, \vec{a})=\sum_{Z^{d}}^{\prime}\left[g^{i j}\left(n_{i}-a_{i}\right)\left(n_{j}-a_{j}\right)\right]^{-s}
$$

(the prime indicates that one should sum over non-zero eigenvalues only).

The generalised Epstein zeta function is difficult to handle explicitly, at least in higher dimensions. Fortunately, we can apply the generalised Poisson resummation formula

$$
\sum_{Z^{d}} \exp \left[-\pi g^{i j}\left(n_{i}-a_{i}\right)\left(n_{j}-a_{j}\right)\right]=\sqrt{\operatorname{det} g_{i j}} \cdot \sum_{Z^{d}} \exp \left[-\pi g_{i j} m^{i} m^{j}-2 \pi i m^{i} a_{i}\right] .
$$

Taking a Mellin transform of the above gives $17 \mid 19$

$$
\zeta_{E}(s, \vec{a})=\frac{\Gamma\left(\frac{d}{2}-s\right)}{\Gamma(s)} \pi^{2 s-\frac{d}{2}} \sqrt{\operatorname{det}\left[g_{i j}\right]} \sum^{\prime}\left(g_{i j} m^{i} m^{j}\right)^{s-\frac{d}{2}} e^{-2 \pi i m^{i} a_{i}} .
$$

The zero-mode, $m_{i}=0$, is eliminated because the zeta function is defined by analytic continuation in $s$. For large $s$ the zero-mode makes no contribution. Equation (8) is a generalisation of the well-known functional equation (reflection formula) for Riemann's (or rather Euler's) zeta function. 20 It is now relatively simple to show that

$$
\begin{aligned}
& \zeta_{E}(0, \vec{a})=0 \\
& \zeta_{E}^{\prime}(0, \vec{a})=\Gamma(d / 2) \pi^{-\frac{d}{2}} \sqrt{\operatorname{det}\left[g_{i j}\right]} \sum_{Z^{d}}^{\prime}\left(g_{i j} n^{i} n^{j}\right)^{-\frac{d}{2}} e^{-2 \pi i n^{j} a_{j}} .
\end{aligned}
$$

The special case $d=2$ has been extensively studied by Kronecker, 21 and for that reason we shall refer to $\zeta_{E}^{\prime}(0, \vec{a})$ as a generalised Kronecker sum. 
Since $\zeta_{E}$ vanishes at the origin the prefactor $(2 \pi / L)^{2}$ in (5) does not contribute to the effective action and

$$
S_{\text {eff }}=\frac{1}{2} \ln \operatorname{det}\left(-D^{2}\right)=-\zeta_{E}^{\prime}(0, \vec{a}) .
$$

Note in particular that in this case the effective action is independent of the renormalization scale $\mu$. This is actually a rather deep result, related to the vanishing of the conformal anomaly for zero electromagnetic field strength. $\frac{16}{16}$ If we consider $\not^{2}$ instead of $-D^{2}$, the only change is to multiply the above with minus one-half the number of spinor components in $d$ dimensions. This general result may be related to known results in one and two dimensions.

In the simplest case, $d=1$, the sum in (9) is a familiar trigonometric series. 22 We find the effective action

$$
S_{\text {eff }}=\frac{1}{2} \ln \operatorname{det}\left(-D^{2}\right)=\ln \left(4 \sin ^{2} \frac{L A}{2}\right) .
$$

Indeed, in one dimension one does not need the reflection formula. The exact zeta function is just a sum of Hurwitz zeta functions and the effective action can be computed directly. Using other techniques, 23 we may show that the effect of a mass term is to change the determinant to

$$
S_{\text {eff }}=\frac{1}{2} \ln \operatorname{det}\left(-D^{2}+m^{2}\right)=\ln \left\{4\left[\sin ^{2}\left(\frac{L A}{2}\right)+\sinh ^{2}\left(\frac{m L}{2}\right)\right]\right\} .
$$

Considering the next simplest case, we observe that in two dimensions any lattice may be re-scaled to satisfy

$$
g=\left[\begin{array}{cc}
1 & \operatorname{Re}(\tau) \\
\operatorname{Re}(\tau) & \tau \bar{\tau}
\end{array}\right]
$$

where $\tau$ is a complex parameter known as the Teichmüller parameter. The Kronecker sum becomes

$$
\zeta_{E}^{\prime}(0, \vec{a})=\frac{\operatorname{Im}(\tau)}{\pi} \sum^{\prime} \frac{e^{-2 \pi i\left(m a_{1}+n a_{2}\right)}}{|m+\tau n|^{2}} .
$$

This sum can be expressed in terms of Riemann theta-functions. ${ }^{17 \mid 24}$ We find the effective action

$$
\zeta_{E}^{\prime}(0, \vec{a})=-2 \log \left|\frac{1}{\eta(\tau)} \quad \vartheta\left[\begin{array}{c}
\frac{1}{2}+a_{1} \\
\frac{1}{2}-a_{2}
\end{array}\right](0 \mid \tau)\right| .
$$

Our theta-function conventions are those of Mumford. 25 This two-dimensional effective action is in fact well known in string theory. 415

Though the discussion has, for clarity, been given in terms of an Abelian gauge potential, the extension to non-Abelian gauge potentials is simple. If the field strength is zero, we may use the non-Abelian version of Stoke's theorem to deduce that the Wilson loops $W(\gamma)=\operatorname{tr}\left(P \exp \oint_{\gamma} \vec{A} \cdot d \vec{x}\right)$ form a representation of $H_{1}$, the first homology group. This implies that the $\vec{A}(x)=\lambda_{a} \vec{A}^{a}(x)$ may be gauge fixed 
to be mutually commuting constant matrices. 9 The results of this section then continue to hold provided one introduces an additional product over the gauge group, $\prod_{a=1}^{\operatorname{dim}(G)}$.

\section{Constant Field Strength: Two Dimensions}

Consider the case of a constant electromagnetic field in two dimensions. Let the area of spacetime be denoted by $S$. The field strength may be written as:

$$
F_{\mu \nu}=\left(\begin{array}{cc}
0 & B \\
-B & 0
\end{array}\right)
$$

As a potential we choose $A_{\mu}=(0, B x)$.

\section{Scalar Particles.}

With this choice the gauged Laplacian appropriate to scalar particles is

$$
-D^{2}=-\partial_{x}^{2}-\left(\partial_{y}-i B x\right)^{2} .
$$

By observing that $-D^{2}$ commutes with the momentum $\hat{P}_{y}=-i \partial_{y}$ we see that on eigenstates of $\hat{P}_{y}$ it reduces to

$$
-D^{2} \rightarrow-\partial_{x}^{2}+B^{2}\left(x-\frac{p}{B}\right)^{2}
$$

Since this is just (twice) the Hamiltonian of a harmonic oscillator it has eigenvalues $\lambda_{p, n}=(2 n+1)|B|$. These eigenvalues are independent of $p$. Thus all levels are degenerate. Later we shall see that this degeneracy is just $2(|B| \cdot S) / 2 \pi$, the prefactor 2 again arising from the sum over both particle and antiparticle states. For notational simplicity take $B$ to be positive.

The zeta function of $-D^{2}+m^{2}$ is given in terms of a Hurwitz zeta function by:

$$
\zeta(s)=\frac{2 B S}{2 \pi} \sum_{n=0}^{\infty}\left[\frac{(2 n+1) B+m^{2}}{\mu^{2}}\right]^{-s} \equiv \frac{2 B S}{2 \pi}\left(\frac{2 B}{\mu^{2}}\right)^{-s} \zeta_{H}\left(s ; \frac{1}{2}+\frac{m^{2}}{2 B}\right) .
$$

The one-loop effective Lagrangian density is $\mathcal{L}_{\text {eff }}=S_{\text {eff }} / S=\frac{1}{2} \ln \operatorname{det}\left(-D^{2}+\right.$ $\left.m^{2}\right) / S=-\frac{1}{2} \zeta^{\prime}(0) / S$. Using properties of the Hurwitz zeta function discussed in the appendix, in particular its value and slope at the point $s=0$, yields:

$$
\mathcal{L}_{\text {eff }}=-\frac{m^{2}}{4 \pi} \cdot \ln \left(\frac{2 B}{\mu^{2}}\right)-\frac{B}{2 \pi} \cdot \ln \left(\Gamma\left(\frac{1}{2}+\frac{m^{2}}{2 B}\right) / \sqrt{2 \pi}\right) .
$$

It is easy to see that the $m \rightarrow 0$ limit is well behaved $-\mathcal{L}_{\text {eff }} \rightarrow(B / 4 \pi) \cdot \ln 2-$ while in the strong-field limit

$$
\mathcal{L}_{\text {eff }}=-\frac{m^{2}}{4 \pi} \cdot \ln \left(\frac{2 B}{\mu^{2}}\right)+\frac{B}{4 \pi} \ln 2+O(1) .
$$

This simple example is already a counterexample to the folk theorem $\mathcal{L}_{\text {eff }} \sim B^{d / 2} \ln B$. 
The weak-field $B \rightarrow 0$ limit may be taken by making use of the doubling formula for the Hurwitz zeta function:

$$
\zeta_{H}\left(s ; \frac{1}{2}+\frac{x}{2}\right)=2^{s} \zeta_{H}(s ; x)-\zeta_{H}\left(s ; \frac{x}{2}\right),
$$

which follows from the definition (A.1) of the appendix. Using this doubling formula we may write (19) as

$$
\begin{aligned}
\zeta(s) & =\frac{2 B S}{2 \pi}\left(\frac{2 B}{\mu^{2}}\right)^{-s} \zeta_{H}\left(\frac{1}{2}+\frac{m^{2}}{2 B}\right) \\
& =\frac{2 B S}{2 \pi}\left[\left(\frac{B}{\mu^{2}}\right)^{-s} \zeta_{H}\left(s ; \frac{m^{2}}{B}\right)-\left(\frac{2 B}{\mu^{2}}\right)^{-s} \zeta_{H}\left(s ; \frac{m^{2}}{2 B}\right)\right] .
\end{aligned}
$$

With the help of equations (A.9), (A.11), and (A.18), we may develop the weakfield expansion for the effective Lagrangian:

[there is a typo, an extaraneous 2 , in the second line of the published version]

$$
\begin{aligned}
\mathcal{L}_{\text {eff }}= & -\frac{1}{2} \frac{\zeta^{\prime}(0)}{S} \\
= & -\frac{B}{2 \pi}\left[-\ln \left(\frac{B}{\mu^{2}}\right) \zeta_{H}\left(0 ; \frac{m^{2}}{B}\right)+\zeta_{H}^{\prime}\left(0 ; \frac{m^{2}}{B}\right)\right. \\
& \left.+\ln \left(\frac{2 B}{\mu^{2}}\right) \zeta_{H}\left(0 ; \frac{m^{2}}{2 B}\right)-\zeta_{H}^{\prime}\left(0 ; \frac{m^{2}}{2 B}\right)\right] \\
= & -\frac{B}{2 \pi}\left[\ln \left(\frac{m^{2}}{\mu^{2}}\right) \zeta_{H}\left(0 ; \frac{m^{2}}{B}\right)+\frac{m^{2}}{B}-\frac{1}{12} \frac{B}{m^{2}}\right. \\
& -\ln \left(\frac{m^{2}}{\mu^{2}}\right) \zeta_{H}\left(0 ; \frac{m^{2}}{2 B}\right)-\frac{1}{2} \frac{m^{2}}{B}+\frac{1}{12} \frac{2 B}{m^{2}} \\
& \left.+\sum_{k=1}^{n-1} \frac{\mathbf{B}_{\mathbf{2 k}+\mathbf{2}}}{(2 k+2)(2 k+1)}\left(\frac{B}{m^{2}}\right)^{2 k+1}\left\{2^{2 k+1}-1\right\}+O\left[\left(\frac{B}{m^{2}}\right)^{n}\right]\right] \\
= & \frac{1}{2 \pi}\left[\frac{1}{2} m^{2}\left\{1-\ln \left(\frac{m^{2}}{\mu^{2}}\right)\right\}+\frac{1}{12} \frac{B^{2}}{m^{2}}\right. \\
& \left.+m^{2} \sum_{k=1}^{n-1} \frac{\mathbf{B}_{\mathbf{2 k}+\mathbf{2}}}{(2 k+2)(2 k+1)}\left(\frac{B}{m^{2}}\right)^{2 k+2}\left\{2^{2 k+1}-1\right\}+O\left[\left(\frac{B}{m^{2}}\right)^{n}\right]\right] .
\end{aligned}
$$

Here the symbol $\mathbf{B}_{\mathbf{n}}$ denotes the $n$th Bernoulli number. Notice that there are no terms logarithmic in $B$ in the weak-field expansion. However, as foreshadowed, the zeta-function renormalization has introduced nonstandard finite terms into the effective Lagrangian. An additional finite (often $\mu$-dependent) renormalization is needed to remove these terms. Removing the constant term in the above equation corresponds to renormalizing the effective cosmological constant, while removing the term proportional to $B^{2}$ corresponds to a finite renormalization of electric charge. (We have chosen our notation in such a manner that electric charge does 
not appear explicitly.) Having implemented these additional finite renormalizations we (finally) display the renormalized effective action as an asymptotic series starting with $B^{4}$ :

$$
\mathcal{L}_{\text {eff }}=\frac{m^{2}}{2 \pi} \sum_{k=1}^{n} \frac{\mathbf{B}_{\mathbf{2 k}+\mathbf{2}}}{(2 k+2)(2 k+1)}\left(\frac{B}{m^{2}}\right)^{2 k+2}\left\{2^{2 k+1}-1\right\}+O\left(B^{2 n+4}\right) .
$$

(We have gone through this simple example in admittedly tedious detail because the same techniques will be used over and over in the following discussion.)

In order to confirm the degeneracy factor we consider the heat kernel of $-D^{2}+$ $m^{2}$. This heat kernel is found to be

$$
\begin{aligned}
K(t) & \equiv \operatorname{tr}^{\prime}\left\{\exp \left(\left[D^{2}-m^{2}\right] t / \mu^{2}\right\}\right. \\
& \equiv e^{-m^{2} t / \mu^{2}} \cdot \sum_{n=0}^{\infty} e^{-\lambda_{n} t / \mu^{2}} \\
& =\frac{B S}{2 \pi} \cdot e^{-m^{2} t / \mu^{2}} \cdot \operatorname{cosech}\left(B t / \mu^{2}\right) .
\end{aligned}
$$

Note that as $t \rightarrow 0, K(t) \rightarrow 2\left(S \mu^{2} / 4 \pi t\right)$, as it should according to the (known) short time behaviour of the heat kernel. This a posteriori proves that we have chosen the correct degeneracy factor $2(B S / 2 \pi)$. Equivalently, one may use the fact that as $B \rightarrow 0$ the heat kernel must approach that of the free two-dimensional diffusion operator, to obtain an alternative verification of the degeneracy factor. It should be emphasized that we are considering the heat kernel appropriate to a complex scalar field. That is to say, the sum in equation (26) includes both particle and anti particle sectors.

Dirac Particles.

The analysis for Dirac spinors closely parallels that of the charged scalar field. The square of the Dirac operator is

$$
\not D^{2}=-D^{2}+\Sigma_{\mu \nu} F^{\mu \nu}=-\partial_{x}^{2}-\left(\partial_{y}-i B x\right)^{2}+\gamma_{5} B .
$$

The eigenvalues are $\lambda_{n}=(2 n+1)|B| \pm B$. We now re-index the states so that the eigenvalues become $\lambda_{n}=2 n|B|$, where the effective degeneracy is $(|B| S / 2 \pi)$ for $n=0$ and $2(|B| S / 2 \pi)$ for $n>0$.

The zeta function of $\not D^{2}$ is given in terms of Hurwitz zeta functions (henceforth we take $B>0$ ),

$$
\begin{aligned}
\zeta(s) & =\frac{B S}{2 \pi}\left\{2 \cdot \sum_{n=1}^{\infty}\left(\frac{2 n B+m^{2}}{\mu^{2}}\right)^{-s}+\left(\frac{m^{2}}{\mu^{2}}\right)^{-s}\right\} \\
& =\frac{B S}{2 \pi} \cdot\left\{2 \cdot\left(\frac{2 B}{\mu^{2}}\right)^{-s} \cdot \zeta_{H}\left(s ; 1+\frac{m^{2}}{2 B}\right)+\left(\frac{m^{2}}{\mu^{2}}\right)^{-s}\right\} \\
& =\frac{B S}{2 \pi} \cdot\left\{2 \cdot\left(\frac{2 B}{\mu^{2}}\right)^{-s} \cdot \zeta_{H}\left(s ; \frac{m^{2}}{2 B}\right)-\left(\frac{m^{2}}{\mu^{2}}\right)^{-s}\right\}
\end{aligned}
$$


The one-loop effective Lagrangian density for spinors is $\mathcal{L}_{\text {eff }}=+\frac{1}{2} \zeta^{\prime}(0) / S$, so that:

$$
\mathcal{L}_{\text {eff }}=\frac{B+m^{2}}{4 \pi} \ln \left(\frac{2 B}{\mu^{2}}\right)+\frac{B}{2 \pi} \ln \Gamma\left(1+\frac{m^{2}}{2 B}\right)-\frac{B}{4 \pi} \ln \left(\frac{2 \pi m^{2}}{\mu^{2}}\right) .
$$

The weak-field limit $B \rightarrow 0$ for spinors is obtained just as it was for scalars. As in the scalar case we must implement an additional finite renormalization of the cosmological constant and the electric charge. Then we obtain

$$
\mathcal{L}_{\text {eff }}=\frac{m^{2}}{4 \pi} \sum_{k=1}^{n} \frac{\mathbf{B}_{\mathbf{2 k}+\mathbf{2}}}{(2 k+2)(2 k+1)}\left(\frac{2 B}{m^{2}}\right)^{2 k+2}+O\left(B^{2 n+4}\right) .
$$

The limit $m \rightarrow 0$ of equation (29) is ill behaved $\left(\mathcal{L}_{\text {eff }} \rightarrow \infty\right)$. This is an infrared singularity associated with the fact that the Dirac operator develops a zero-mode as $m \rightarrow 0$. One may deal with the zero-mode by simply dropping the ground state $(n=0)$ from the sum prior to taking the $m \rightarrow 0$ limit. Equivalently, for massless Dirac spinors one must exclude the zero mode "by hand". Recall that the definition of the zeta function is in terms of $\sum^{\prime}$ not $\sum$. The difference has up to now been irrelevant. Taking careful note of this difference leads to:

$$
\zeta(s)=\frac{2 B S}{2 \pi} \sum_{n=1}^{\infty}\left(\frac{2 n B}{\mu^{2}}\right)^{-s}=\frac{2 B S}{2 \pi}\left(\frac{2 B}{\mu^{2}}\right)^{-s} \cdot \zeta_{R}(s) .
$$

The one-loop effective Lagrangian density is simplified to

$$
\mathcal{L}_{\text {eff }}=+\frac{B}{2 \pi} \cdot \ln \left(\frac{B}{\pi \mu^{2}}\right) .
$$

The heat kernel of $\not D^{2}+m^{2}$ is readily evaluated

$$
K(t)=\frac{B S}{2 \pi} \operatorname{coth}\left(\frac{B t}{\mu^{2}}\right) \exp \left(-\frac{m^{2} t}{\mu^{2}}\right) .
$$

Note that as $t \rightarrow 0$ that $K(t) \rightarrow 2 \cdot\left(S \mu^{2} / 4 \pi t\right)$, as indeed it should. (The factor of 2 reflects the existence of two spinor components in two dimensions). In the massless case the "zero-mode-suppressed" heat kernel is

$$
K^{\prime}(t)=\frac{B S}{2 \pi}\left[\operatorname{coth}\left(\frac{B t}{\mu^{2}}\right)-1\right] .
$$

Note that $K^{\prime}(t) \rightarrow 0$ as $t \rightarrow \infty$, thanks to the explicit exclusion of the zero mode.

Finally, note that calculation of the heat kernels allows one to deduce all the Seeley-deWitt coefficients for the case of constant field. Use of the Taylor series for $\operatorname{cosech}(B t)$ and $\operatorname{coth}(B t)$ yields:

$$
\begin{aligned}
& a_{2 n}\left(-D^{2}\right)=-2 \frac{\left(2^{2 n-1}-1\right)}{(2 n) !} \cdot \mathbf{B}_{\mathbf{2 n}} \cdot B^{2 n}, \\
& a_{2 n}\left(D^{2}\right)=\frac{\left(2^{2 n+1}\right)}{(2 n) !} \cdot \mathbf{B}_{\mathbf{2 n}} \cdot B^{2 n}
\end{aligned}
$$


where $\mathbf{B}_{\mathbf{n}}$ denotes the $n$th Bernoulli number. The 'odd' coefficients $a_{2 n+1}$ all vanish. It is very unusual to know all the $a_{n}$. For arbitrary fields these coefficients are leading order terms in a low-momentum approximation. Thus they remain interesting for general fields.

\section{Constant Field Strength: Three Dimensions}

In three dimensions the field strength may be written as:

$$
F_{\mu \nu}=\left(\begin{array}{ccc}
0 & 0 & 0 \\
0 & 0 & B \\
0 & -B & 0
\end{array}\right) .
$$

Let the volume of spacetime be denoted by $V=S \cdot L$. In the gauge $A_{\mu}=(0,0, B y)$, we may immediately write down the three-dimensional heat kernel $K_{d=3}(t)=\frac{\mu L}{\sqrt{4 \pi t}}$. $K_{d=2}(t)$. Three-dimensional zeta functions quickly follow:

$$
\begin{aligned}
\zeta_{d=3}(s) & =\frac{1}{\Gamma(s)} \cdot \int_{0}^{\infty} t^{s-1} K_{d=3}(t) \\
& =\frac{\mu L}{\sqrt{4 \pi}} \cdot \frac{\Gamma\left(s-\frac{1}{2}\right)}{\Gamma(s)} \cdot \zeta_{d=2}\left(s-\frac{1}{2}\right) .
\end{aligned}
$$

All factors are analytic at $s=0$. In particular, since $\frac{1}{\Gamma(s)} \sim s+0\left(s^{2}\right)$, the derivative at $s=0$ is

$$
\zeta_{d=3}^{\prime}(0)=\frac{\mu L}{\sqrt{4 \pi}} \cdot \Gamma\left(-\frac{1}{2}\right) \cdot \zeta_{d=2}\left(-\frac{1}{2}\right)=-\mu L \cdot \zeta_{d=2}\left(-\frac{1}{2}\right) .
$$

This means that the one-loop effective Lagrangian densities are given in terms of $\zeta_{H}\left(-\frac{1}{2}, x\right)$. Using results of the two-dimensional discussion we see:

$$
\begin{array}{ll}
\text { Scalar Particles: } & \mathcal{L}_{\text {eff }}=\frac{1}{4 \pi} \cdot(2 B)^{3 / 2} \cdot \zeta_{H}\left(-\frac{1}{2} ; \frac{1}{2}+\frac{m^{2}}{2 B}\right), \\
\text { Dirac Particles: } & \mathcal{L}_{\text {eff }}=-\frac{1}{8 \pi} \cdot 2 B \cdot\left\{2 \sqrt{2 B} \zeta_{H}\left(-\frac{1}{2} ; 1+\frac{m^{2}}{2 B}\right)+m\right\} .
\end{array}
$$

Note that these effective actions are independent of the renormalization scale $\mu$. This (nonobvious) result is a consequence of the vanishing of the conformal anomaly in odd-dimensional space-times. For strong fields, the Hurwitz zeta function can be computed by convergent series. [See equation (A.6) of the appendix.] For the cases of interest:

$$
\begin{aligned}
& \zeta_{H}\left(-\frac{1}{2} ; \frac{1}{2}+\frac{m^{2}}{2 B}\right)=\zeta_{H}\left(-\frac{1}{2} ; \frac{1}{2}\right)-\sum_{l=1}^{\infty}(-)^{l} \frac{(2 l-3) ! !}{2^{l} l !} \cdot\left(\frac{m^{2}}{2 B}\right)^{l} \cdot \zeta_{H}\left(-\frac{1}{2}+l ; \frac{1}{2}\right), \\
& \zeta_{H}\left(-\frac{1}{2} ; 1+\frac{m^{2}}{2 B}\right)=\zeta_{R}\left(-\frac{1}{2}\right)-\sum_{l=1}^{\infty}(-)^{l} \frac{(2 l-3) ! !}{2^{l} l !} \cdot\left(\frac{m^{2}}{2 B}\right)^{l} \cdot \zeta_{R}\left(-\frac{1}{2}+l\right) .
\end{aligned}
$$

The coefficients in these expansions can be obtained (with severely limited accuracy) from the tables of Jahnke and Emde. $\frac{26}{26}$ Alternatively, the proliferation of personal 
computers allows these coefficients to easily be computed to any desired accuracy. Note the presence of fractional powers of the field strength and the absence of logarithmic terms when equation (41) is substituted into (40) in order to obtain an expansion for the effective Lagrangian. We shall see that these general features persist in any odd number of dimensions, at least for constant field strength. The leading strong-field limit in three dimensions is

$$
\begin{array}{ll}
\text { Scalar particles: } & \mathcal{L}_{\text {eff }}=\frac{1}{4 \pi}(2 B)^{3 / 2} \zeta_{H}\left(-\frac{1}{2} ; \frac{1}{2}\right) . \\
\text { Spinor particles: } & \mathcal{L}_{\text {eff }}=-\frac{1}{2 \pi}(2 B)^{3 / 2} \zeta_{R}\left(-\frac{1}{2}\right) .
\end{array}
$$

The leading term in the strong-field expansion for the spinor Lagrangian has previously been obtained by Redlich. 27

The leading coefficients appearing in the strong-field limit have been evaluated numerically. This was done by using the reflection formula for the Riemann zeta function to write $\zeta_{H}\left(-\frac{1}{2} ; 1\right)=\zeta_{R}\left(-\frac{1}{2}\right)=-\frac{1}{4 \pi} \zeta_{R}\left(\frac{3}{2}\right)$. Note that $\zeta_{R}\left(\frac{3}{2}\right)$ is given by a nicely convergent series suitable for computer evaluation. We find $\zeta_{R}\left(\frac{3}{2}\right) \approx$ $2.612375, \zeta_{R}\left(-\frac{1}{2}\right) \approx-0.207886$. In a similar vein, we use the "doubling formula", $\zeta_{H}\left(s ; \frac{1}{2}\right)=\left(2^{s}-1\right) \zeta_{R}(s)$ to deduce

$$
\zeta_{H}\left(-\frac{1}{2} ; \frac{1}{2}\right)=\frac{1}{4 \pi}\left\{1-\frac{1}{\sqrt{2}}\right\} \zeta_{R}\left(\frac{3}{2}\right) \approx 0.060888 .
$$

In order to obtain the weak-field limit for the effective Lagrangian, we return to equation (40). We shall need equation (A.7) of the appendix, evaluated at $s=\frac{1}{2}$.

$$
\begin{aligned}
\zeta_{H}\left(-\frac{1}{2} ; x\right)= & \frac{1}{\Gamma\left(-\frac{1}{2}\right)}\left[x^{3 / 2} \Gamma\left(-\frac{3}{2}\right)+\frac{1}{2} x^{1 / 2} \Gamma\left(-\frac{1}{2}\right)\right. \\
& \left.+\sum_{k=1}^{n} \mathbf{B}_{\mathbf{2 k}} \frac{\Gamma\left(2 k-\frac{3}{2}\right)}{(2 k) !} x^{3 / 2-2 k}+O\left(x^{1 / 2-2 n}\right)\right]
\end{aligned}
$$

After an additional finite renormalization of the cosmological constant and the electric charge, the weak-field limit for the scalar effective Lagrangian is

$$
\mathcal{L}_{\text {eff }}=-\frac{m^{3}}{\pi} \sum_{k=1}^{n} \mathbf{B}_{\mathbf{2 k}+\mathbf{2}} \frac{\Gamma\left(2 k+\frac{1}{2}\right)}{(2 k+2) ! \Gamma\left(-\frac{1}{2}\right)}\left[2^{2 k}-\frac{1}{2}\right]\left(\frac{B}{m^{2}}\right)^{2 k+2}+O\left(B^{2 n+4}\right),
$$

while the spinor Lagrangian is

$$
\mathcal{L}_{\text {eff }}=-\frac{m^{3}}{4 \pi} \sum_{k=1}^{n} \mathbf{B}_{\mathbf{2 k}+\mathbf{2}} \frac{\Gamma\left(2 k+\frac{1}{2}\right)}{(2 k+2) ! \Gamma\left(-\frac{1}{2}\right)}\left(\frac{2 B}{m^{2}}\right)^{2 k+2}+O\left(B^{2 n+4}\right) .
$$

Only even, positive-integer powers of the field enter the weak-field expansion.

Notice the absence form our discussion of the Chern-Simons secondary characteristic class (the topological mass term for the photon). This term might be expected to appear on rather general grounds, following arguments of Niemi and 
Semenoff 28 and Redlich 27. However, a simple analysis is sufficient to show that the Chern-Simons term vanishes for constant field strength. $27 / 29$ We should also note the appearance of fractional powers of the field strength has also been noted in Redlich's calculation for a massive spinor. 29 The present calculation generalizes this result to the massive case and also to scalar particles.

\section{Constant Field Strength: Four Dimensions}

In four dimensions the field strength may be written as:

$$
F_{\mu \nu}=\left(\begin{array}{cccc}
0 & E & 0 & 0 \\
-E & 0 & 0 & 0 \\
0 & 0 & 0 & B \\
0 & 0 & -B & 0
\end{array}\right)
$$

so that the four-dimensional problem breaks up into two two-dimensional ones.

This result follows from the block-diagonalizability of antisymmetic matrices through orthogonal transformations. It is important to know that $E$ and $B$ are invariant scalars that characterise the electromagnetic field. Note that $E^{2}+B^{2}=$ $\frac{1}{2} F_{\mu \nu} F^{\mu \nu}=\frac{1}{2} F^{2}$, and $2 E B=\epsilon_{\mu \nu \sigma \rho} F^{\mu \nu} F^{\sigma \rho}=F \tilde{F}$. While it is more common to characterise the field in terms of $F^{2}$ and $F \tilde{F}$, it is more useful for us to use the invariants $E$ and $B$. Though we shall be working in Euclidean space, we note that the result (47) also obtains in Minkowski space. This follows from the fact that it is always possible to make a Lorentz boost such the electric and magnetic fields become parallel and then (by a rotation) to make both fields point in the $x$ direction.

\section{1. $E=0, B \neq 0$}

Our analysis in this case parallels that of three dimensions. Writing the volume of spacetime as $\Omega=\Omega_{\perp} \cdot S$, and choosing the gauge as $A_{\mu}=(0,0,0, B y)$, we factorize the heat kernel

$$
K_{d=4}(t)=D \frac{\Omega_{\perp} \mu^{2}}{4 \pi t} \cdot K_{d=2}(t) .
$$

Here $D=1$ for scalars and $D=2$ for spinors, reflecting the fact that fourdimensional spinors possess twice as many degrees of freedom as two-dimensional spinors. The four-dimensional zeta function is given by

$$
\zeta_{d=4}(s)=\frac{D}{\Gamma(s)} \cdot \int_{0}^{\infty} t^{s-1} K_{d=4}(t) d t=D \frac{\Omega_{\perp} \mu^{2}}{4 \pi} \cdot \frac{\zeta_{d=2}(s-1)}{s-1} .
$$

Inserting the results of the two-dimensional case yields

$$
\text { Scalar Particles: } \quad \zeta(s)=\frac{B^{2} \Omega}{2 \pi^{2}} \cdot\left(\frac{2 B}{\mu^{2}}\right)^{-s} \cdot \frac{\zeta_{H}\left(s-1 ; \frac{1}{2}+\frac{m^{2}}{2 B}\right)}{(s-1)},
$$




$$
\text { Dirac Particles: } \quad \begin{aligned}
\zeta(s)= & \frac{B^{2} \Omega}{\pi^{2}} \cdot\left(\frac{2 B}{\mu^{2}}\right)^{-s} \cdot \frac{\zeta_{H}\left(s-1 ; 1+\frac{m^{2}}{2 B}\right)}{(s-1)} \\
& +\frac{m^{2} B \Omega}{4 \pi^{2}}\left\{\frac{(m / \mu)^{-2 s}}{s-1}\right\}
\end{aligned}
$$

Taking derivatives at $s=0$ is straightforward.

\subsubsection{Scalar Particles:}

For the effective Lagrangian density one obtains:

$$
\mathcal{L}_{\text {eff }}=\frac{B^{2}}{4 \pi^{2}}\left\{\left[1-\ln \left(\frac{2 B}{\mu^{2}}\right)\right] \zeta_{H}\left(-1 ; \frac{1}{2}+\frac{m^{2}}{2 B}\right)+\zeta_{H}^{\prime}\left(-1 ; \frac{1}{2}+\frac{m^{2}}{2 B}\right)\right\} .
$$

Using results from the appendix one finds that

$$
\begin{aligned}
\mathcal{L}_{\text {eff }}=\frac{1}{4 \pi^{2}}\left[\left(\frac{B^{2}}{24}-\frac{m^{4}}{8}\right)\right. & \cdot\left[1-\ln \left(\frac{2 B}{\mu^{2}}\right)\right]+B^{2} \zeta_{H}^{\prime}\left(-1 ; \frac{1}{2}\right)+\frac{m^{4}}{8} \\
+ & \left.B^{2} \int_{0}^{\frac{m^{2}}{2 B}} \ln \left(\Gamma\left(\frac{1}{2}+y\right) / \sqrt{2 \pi}\right) d y .\right]
\end{aligned}
$$

This form makes it easy to extract the large field limit $B \gg m^{2}$,

$\mathcal{L}_{\text {eff }}=\frac{1}{4 \pi^{2}}\left[\left(\frac{B^{2}}{24}-\frac{m^{4}}{8}\right) \cdot\left[1-\ln \left(\frac{2 B}{\mu^{2}}\right)\right]+B^{2} \zeta_{H}^{\prime}\left(-1 ; \frac{1}{2}\right)+\frac{m^{4}}{8}-\frac{B m^{2}}{4} \ln 2\right]$.

The coefficient $\zeta_{H}^{\prime}\left(-1 ; \frac{1}{2}\right)$ has been evaluated numerically. This was done by noting that $\zeta_{H}\left(s ; \frac{1}{2}\right)=\left(2^{s}-1\right) \cdot \zeta_{R}(s)$; and by using the reflection formula (see appendix) to relate $\zeta_{H}^{\prime}\left(-1 ; \frac{1}{2}\right)$ to $\zeta_{R}^{\prime}(+2)$. We obtain $\zeta_{H}^{\prime}\left(-1 ; \frac{1}{2}\right) \approx 0.053829$.

In order to obtain the weak-field limit it is easiest to return to equation (51) and to use the doubling formula (22) along with equations (A.10) and (A.16) of the appendix. This procedure yields an asymptotic expansion in terms of powers of $B^{2}$; in particular there are no terms logarithmic in field strength. After performing the usual additional finite renormalization of cosmological constant and electric charge, we obtain the extended Euler-Heisenberg effective Lagrangian

$\mathcal{L}_{\text {eff }}=\frac{B^{2}}{16 \pi^{2}}\left[\frac{-7}{360} \frac{B^{2}}{m^{4}}+\sum_{k=1}^{n} \frac{\mathbf{B}_{\mathbf{2 k}+\mathbf{2}}}{(2 k+2)(2 k+1)(2 k)}\left[2^{2 k+2}-2\right]\left(\frac{B}{m^{2}}\right)^{2 k}+O\left(B^{2 n+2}\right)\right]$.

This result should be compared with the Minkowski-space Euler-Heisenberg Lagrangian obtained by Schwinger. ${ }^{3}$ In order to make the comparison, we must take Schwinger's formula and after setting $E=0$ multiply by -1 . In the general case, for which $E$ does not vanish, we must replace the $E$ 's in Schwinger's formula with $i E$ 
before multiplying by -1 to obtain the Euclidean result. Finally, we have implicitly chosen units for the renormalized charge so that Schwinger's $\alpha^{2}$ corresponds to our $1 / 16 \pi^{2}$. After making these adjustments for notation and metric signature, we may confirm that our expansion agrees with Schwinger to the leading order (which is the only order explicitly displayed in reference 8 . Note though, that in this zeta function formalism, it is easy to display all orders of the asymptotic expansion for the Euler-Heisenberg Lagrangian in the weak-field limit.

\subsubsection{Dirac Particles:}

For Dirac spinors one obtains

$$
\begin{gathered}
\mathcal{L}_{\text {eff }}=-\frac{B^{2}}{2 \pi^{2}}\left\{\left[1-\ln \left(\frac{2 B}{\mu^{2}}\right)\right] \zeta_{H}\left(-1 ; 1+\frac{m^{2}}{2 B}\right)+\zeta_{H}^{\prime}\left(-1 ; 1+\frac{m^{2}}{2 B}\right)\right\} \\
+\frac{m^{2} B}{8 \pi^{2}}\left[\ln \left(\frac{m^{2}}{\mu^{2}}\right)-1\right] .
\end{gathered}
$$

A little work using results given in the appendix yields

$$
\begin{gathered}
\mathcal{L}_{\text {eff }}=\frac{1}{2 \pi^{2}}\left\{\left(\frac{B^{2}}{12}+\frac{m^{2} B}{4}+\frac{m^{4}}{8}\right)\left[1-\ln \left(\frac{2 B}{\mu^{2}}\right)\right]-B^{2} \zeta_{R}^{\prime}(-1)-\frac{m^{4}}{8}-\frac{m^{2} B}{4}\right. \\
\left.+\frac{m^{2} B}{4}\left[\ln \left(\frac{m^{2}}{\mu^{2}}\right)-1\right]-B^{2} \int_{0}^{\frac{m^{2}}{2 B}} \ln (\Gamma(1+y) / \sqrt{2 \pi}) d y\right\}
\end{gathered}
$$

For strong fields one uses $\ln \Gamma(1+\epsilon)=-\gamma \epsilon+O\left(\epsilon^{2}\right)$, to establish

$$
\begin{aligned}
\mathcal{L}_{\text {eff }}=\frac{1}{2 \pi^{2}} & \left\{\left(\frac{B^{2}}{12}+\frac{m^{2} B}{4}+\frac{m^{4}}{8}\right)\left[1-\ln \left(\frac{2 B}{\mu^{2}}\right)\right]-B^{2} \zeta_{R}^{\prime}(-1)-\frac{m^{4}}{8}-\frac{m^{2} B}{4}\right. \\
& \left.+\frac{m^{2} B}{4}\left[\ln \left(\frac{m^{2}}{\mu^{2}}\right)-1\right]+\frac{m^{2} B}{4} \ln (2 \pi)+\frac{\gamma}{8} m^{4}\right\}+m^{4} O\left(m^{2} / B\right) .
\end{aligned}
$$

The coefficient $\zeta_{R}^{\prime}(-1) \approx-0.165421$ has been calculated numerically.

In order to obtain the weak-field limit we return to equation (55) and employ the identity $\zeta_{H}(s ; 1+x)=\zeta_{H}(s ; x)-x^{-s}$. Just as in the scalar case we obtain an (extended) Euler-Heisenberg Lagrangian.

$$
\mathcal{L}_{\text {eff }}=\frac{B^{2}}{16 \pi^{2}}\left[\frac{-2}{45} \frac{B^{2}}{m^{4}}+\sum_{k=1}^{n} \frac{4 \mathbf{B}_{\mathbf{2 k}+\mathbf{2}}}{(2 k+2)(2 k+1)(2 k)}\left(\frac{2 B}{m^{2}}\right)^{2 k}+O\left(B^{2 n+2}\right)\right],
$$

which specializes to the Euclidean version of Schwinger's result. 8

The results of this section may easily be extended to discuss the case $B=0$, $E \neq 0$. One needs merely replace $B$ by $E$ in all formulae to obtain valid Euclidean space results. Recall, though, that in continuing to Minkowski space one effects 
the transformation $E \rightarrow i E$. In the next section we shall begin to address the complexities encountered when $E$ and $B$ are both nonzero.

\section{2. $E \neq 0, B \neq 0$}

The scalar spectrum is given by $\lambda=(2 n+1)|E|+\left(2 n^{\prime}+1\right)|B|+m^{2}$, where all the states have the degeneracy $2(|E B| \Omega) / 4 \pi^{2}$. The zeta function is

$$
\zeta(s)=\frac{|E B| \Omega}{2 \pi^{2}} \cdot \sum_{n, n^{\prime}=0}^{\infty}\left[\frac{(2 n+1)|E|+\left(2 n^{\prime}+1\right)|B|+m^{2}}{\mu^{2}}\right]^{-s} .
$$

For spinors the analogous zeta function is a trifle more complicated [the published version of this equation has a typo]:

$$
\begin{aligned}
\zeta(s)=\frac{|E B| \Omega}{4 \pi^{2}} \cdot\{ & 4 \cdot \sum_{n, n^{\prime}=1}^{\infty}\left[\frac{2 n|E|+2 n^{\prime}|B|+m^{2}}{\mu^{2}}\right]^{-s} \\
& +2 \cdot \sum_{n=1}^{\infty}\left[\frac{2 n|E|+m^{2}}{\mu^{2}}\right]^{-s} \\
& +2 \cdot \sum_{n^{\prime}=1}^{\infty}\left[\frac{2 n^{\prime}|B|+m^{2}}{\mu^{2}}\right]^{-s} \\
+1 & \left.\cdot\left[\frac{m^{2}}{\mu^{2}}\right]^{-s}\right\} .
\end{aligned}
$$

These zeta functions are in general so unwieldy as to be unmanageable. To proceed we restrict ourselves to the case $|E|=|B|=F$, corresponding to self-dual and antiself-dual constant fields. In this special situation we can recast these zeta functions in terms of the elementary Hurwitz zeta functions.

For the case of a scalar particle

$$
\zeta(s)=\frac{F^{2} \Omega}{2 \pi^{2}} \cdot\left(\frac{2 F}{\mu^{2}}\right)^{-s} \cdot \sum_{n, n^{\prime}=0}^{\infty}\left(n+n^{\prime}+1+\frac{m^{2}}{2 F}\right)^{-s}
$$

We define $t=n+n^{\prime}$, and observe that there are precisely $t+1$ ways in which $n$ and $n^{\prime}$ can be arranged to sum to $t$, consequently

$$
\begin{aligned}
\zeta(s) & =\frac{F^{2} \Omega}{2 \pi^{2}} \cdot\left(\frac{2 F}{\mu^{2}}\right)^{-s} \cdot \sum_{t=0}^{\infty}(t+1) \cdot\left(t+1+\frac{m^{2}}{2 F}\right)^{-s} \\
& =\frac{F^{2} \Omega}{2 \pi^{2}} \cdot\left(\frac{2 F}{\mu^{2}}\right)^{-s} \cdot\left\{\zeta_{H}\left(s-1 ; 1+\frac{m^{2}}{2 F}\right)-\frac{m^{2}}{2 F} \cdot \zeta_{H}\left(s ; 1+\frac{m^{2}}{2 F}\right)\right\} \\
& =\frac{F^{2} \Omega}{2 \pi^{2}} \cdot\left(\frac{2 F}{\mu^{2}}\right)^{-s} \cdot\left\{\zeta_{H}\left(s-1 ; \frac{m^{2}}{2 F}\right)-\frac{m^{2}}{2 F} \cdot \zeta_{H}\left(s ; \frac{m^{2}}{2 F}\right)\right\} .
\end{aligned}
$$


Here we have used $\zeta_{H}(s ; 1+x)=\zeta_{H}(s ; x)-x^{-s}$. For the Dirac spinor, very similar results obtain, though one must be careful with degeneracy factors:

$$
\begin{aligned}
\zeta(s)=\frac{F^{2} \Omega}{4 \pi^{2}}\{ & \left(\frac{2 F}{\mu^{2}}\right)^{-s} \cdot 4 \sum_{n, n^{\prime}=1}^{\infty}\left(n+n^{\prime}+\frac{m^{2}}{2 F}\right)^{-s} \\
& \left.+\left(\frac{2 F}{\mu^{2}}\right)^{-s} \cdot 2 \cdot 2 \sum_{n=1}^{\infty}\left(n+\frac{m^{2}}{2 F}\right)^{-s}+\left(\frac{m^{2}}{\mu^{2}}\right)^{-s}\right\} .
\end{aligned}
$$

Re-indexing the sum, using $t=n+n^{\prime}-1$, yields

$$
\zeta_{\text {Spinor }}(s)=2 \zeta_{\text {Scalar }}(s)+\frac{F^{2} \Omega}{4 \pi^{2}}\left(\frac{m^{2}}{\mu^{2}}\right)^{-s}
$$

Apart from the ground-state contribution $\left(n=n^{\prime}=0 ; \lambda=m^{2}\right)$ the scalar and spinor zeta functions are proportional to one another. This desirable property does not, unfortunately, continue to hold if $|E| \neq|B|$. To discuss the effective action, the strong-field limit, and the weak-field limit, it suffices to discuss the scalars.

The effective Lagrangian for scalars is

$$
\begin{aligned}
\mathcal{L}_{\text {eff }}=\frac{F^{2}}{4 \pi^{2}}\left\{\ln \left(\frac{2 F}{\mu^{2}}\right)\right. & {\left[\zeta_{H}\left(-1 ; \frac{m^{2}}{2 F}\right)-\frac{m^{2}}{2 F} \zeta_{H}\left(0 ; \frac{m^{2}}{2 F}\right)\right] } \\
& \left.-\zeta_{H}^{\prime}\left(-1 ; \frac{m^{2}}{2 F}\right)+\frac{m^{2}}{2 F} \zeta_{H}^{\prime}\left(0 ; \frac{m^{2}}{2 F}\right)\right\} .
\end{aligned}
$$

Using results from the appendix yields

$$
\begin{aligned}
\mathcal{L}_{\text {eff }}= & -\frac{1}{4 \pi^{2}}\left[\frac{1}{12} F^{2}-\frac{1}{8} m^{4}\right] \ln \left(\frac{2 F}{\mu^{2}}\right) \\
& -\frac{F^{2}}{4 \pi^{2}} \cdot \zeta_{R}^{\prime}(-1) \\
& -\frac{1}{4 \pi^{2}}\left\{\frac{1}{4} m^{2} F+\frac{1}{8} m^{4}-\frac{1}{2} m^{2} F \ln \left(\frac{\Gamma\left(1+\frac{m^{2}}{2 F}\right)}{\sqrt{2 \pi}}\right)\right\} \\
& -\frac{F^{2}}{4 \pi^{2}} \cdot \int_{0}^{\frac{m^{2}}{2 F}} \ln (\Gamma(1+y) / \sqrt{2 \pi}) d y .
\end{aligned}
$$

The strong-field and weak-field limits may be written down in the standard manner, we omit details and present the results. For strong fields

$$
\begin{aligned}
\mathcal{L}_{\text {eff }}=-\frac{1}{4 \pi^{2}}\{ & {\left[\frac{1}{12} F^{2}-\frac{1}{8} m^{4}\right] \ln \left(\frac{2 F}{\mu^{2}}\right)+F^{2} \cdot \zeta_{R}^{\prime}(-1) } \\
& \left.+\frac{1}{4} m^{2} F+\frac{1}{8}(1+\gamma) m^{4}+O\left(\frac{m^{6}}{F}\right)\right\} .
\end{aligned}
$$


For weak fields, the Euclidean-space (scalar) Euler-Heisenberg Lagrangian is

$$
\mathcal{L}_{\text {eff }}=\frac{F^{2}}{16 \pi^{2}}\left[\frac{-1}{15} \frac{F^{2}}{m^{4}}+\sum_{k=1}^{n} \frac{\mathbf{B}_{\mathbf{2 k}+\mathbf{2}}}{(2 k+2)(2 k+1)}\left\{4+\frac{2}{k}\right\}\left(\frac{2 F}{m^{2}}\right)^{2 k}+O\left(F^{2 n+2}\right)\right] .
$$

When continued to Minkowski space, the lowest term in this expansion reproduces

Schwinger's result. ${ }^{8}$ To relate this scalar result to the case of a massive Dirac spinor, one notes that

$$
\mathcal{L}_{\text {Spinor }}=-2 \cdot \mathcal{L}_{\text {Scalar }}+\frac{F^{2}}{8 \pi^{2}} \cdot \ln \left(m^{2} / \mu^{2}\right)
$$

The massless case affords considerable simplifications; for the scalar one obtains

$$
\zeta(s)=\frac{F^{2} \Omega}{2 \pi^{2}} \cdot\left(\frac{2 F}{\mu^{2}}\right)^{-s} \cdot \zeta_{R}(s-1) .
$$

This leads to an effective action

$$
\mathcal{L}_{\text {eff }}=-\frac{F^{2}}{4 \pi^{2}} \cdot\left[\frac{1}{12} \ln \left(\frac{2 F}{\mu^{2}}\right)+\zeta_{R}^{\prime}(-1)\right] .
$$

The term proportional to $F^{2}$ is an artifact of the zeta-function method, and may, in the usual fashion, be removed through a finite renormalization of the electric charge. For massless Dirac spinors, explicit exclusion of the zero-mode leads to $\mathcal{L}_{\text {Spinor }}=-2 \mathcal{L}_{\text {Scalar }}$. These massless (anti-)self-dual effective actions have been previously discussed in the literature, see for example 15130131 .

\section{Even Dimensionality}

In $d=2 N$ dimensions a constant field may be brought into the block diagonal form

$$
F_{\mu \nu}=\left(\begin{array}{cccccc}
0 & B_{1} & & & \\
-B_{1} & 0 & & & \\
& & \cdot & & & \\
& & \cdot & & \\
& & \cdot & & \\
& & & 0 & B_{N} \\
& & & -B_{N} & 0
\end{array}\right),
$$

where the $\pm B_{j}$ are the zeros of the characteristic polynomial $\operatorname{det}(B \cdot I+i F)$. The problem thus decomposes into $N$ two-dimensional problems, allowing the associated zeta functions to be written down by inspection.

Scalar Particles

The eigenspectrum is $\lambda_{\vec{n}}=\left\{\sum_{i=1}^{N}\left(2 n_{i}+1\right)\left|B_{i}\right|\right\}+m^{2}$ with degeneracy $d_{n}=$ $\prod_{i=1}^{N} 2\left(\left|B_{i}\right| \cdot S_{i} / 2 \pi\right)$. The zeta function is

$$
\zeta(s)=2 \prod_{i=1}^{N}\left(\frac{\left|B_{i}\right| S_{i}}{2 \pi}\right) \cdot \sum_{n_{i}=0}^{\infty}\left(\frac{\left\{\sum_{i=1}^{N}\left(2 n_{i}+1\right)\left|B_{i}\right|\right\}+m^{2}}{\mu^{2}}\right)^{-s}
$$




$$
=2 \frac{\Omega}{\Gamma(s)} \cdot \int_{0}^{\infty} t^{s-1}\left\{\prod_{i=1}^{N}\left(\frac{\left|B_{i}\right|}{4 \pi}\right) \operatorname{cosech}\left(\frac{\left|B_{i}\right| t}{\mu^{2}}\right)\right\} \exp \left(-\frac{m^{2} t}{\mu^{2}}\right) .
$$

Dirac Particles

The eigenspectrum is $\lambda_{\vec{n}}=\left\{\sum_{i=1}^{N} 2 n_{i}\left|B_{i}\right|\right\}+m^{2}$ with degeneracy $d_{n}=\prod_{i=1}^{N}\left(\left|B_{i}\right|\right.$. $\left.S_{i} / 2 \pi\right) \cdot 2^{\omega\left(n_{i}\right)}$, where $\omega(0)=0$, and $\omega(n>0)=1$. The zeta function is

$$
\begin{aligned}
\zeta(s) & =\prod_{i=1}^{N}\left(\frac{\left|B_{i}\right| S_{i}}{2 \pi}\right) \cdot \sum_{n_{i}=0}^{\infty} 2^{\omega(\vec{n})}\left(\frac{\left\{\sum_{i=1}^{N} 2 n_{i}\left|B_{i}\right|\right\}+m^{2}}{\mu^{2}}\right)^{-s} \\
& =\frac{\Omega}{\Gamma(s)} \cdot \int_{0}^{\infty} t^{s-1}\left\{\prod_{i=1}^{N}\left(\frac{\left|B_{i}\right|}{2 \pi}\right) \operatorname{coth}\left(\frac{\left|B_{i}\right| t}{\mu^{2}}\right)\right\} \exp \left(-\frac{m^{2} t}{\mu^{2}}\right) .
\end{aligned}
$$

These zeta functions are tedious to calculate with in general, to proceed we make the radically simplifying assumptions that $\left|B_{1}\right|=\left|B_{2}\right|=\ldots=B$, and that $m=0$. Of course, in two dimensions the condition on the field is always met since there is only one $B$. In four dimensions it means that we confine ourselves to (anti-)selfdual backgrounds. With these assumptions the scalar zeta function is simplified to

$$
\zeta(s)=\left(\frac{B S}{4 \pi}\right)^{N} \cdot \frac{\left(B / \mu^{2}\right)^{-s}}{\Gamma(s)} \cdot \int_{0}^{\infty} t^{s-1}(\operatorname{cosech} t)^{N}
$$

while for the massless spinor, exclusion of the zero mode leads to

$$
\zeta(s)=\left(\frac{B S}{2 \pi}\right)^{N} \cdot \frac{\left(B / \mu^{2}\right)^{-s}}{\Gamma(s)} \cdot \int_{0}^{\infty} t^{s-1}\left\{(\operatorname{coth} t)^{N}-1\right\} .
$$

Note that for $N=2$, (i.e., four dimensions), the spinorial zeta function is four times the scalar zeta function, in agreement with our earlier result.

For these backgrounds we can relate the zeta function in $d$-dimensions to the ones in $(d-2)$ and $(d-4)$ dimensions by recursion relations. For the scalar case, a repeated integration by parts establishes

$$
\zeta_{N}(s)=\left(\frac{\mu^{2} S}{4 \pi}\right)^{2} \cdot \frac{\zeta_{N-2}(s-2)}{(N-1)(N-2)}-\left(\frac{B S}{4 \pi}\right)^{2} \cdot\left(\frac{N-2}{N-1}\right) \cdot \zeta_{N-2}(s),
$$

so that for the scalar there are two disconnected series starting with $d=2, d=4$, respectively. For the spinorial case a single integration by parts suffices to obtain

$$
\zeta_{N}(s)=\frac{\mu^{2} S}{2 \pi} \cdot \frac{\zeta_{N-1}(s-1)}{N-1}+\left(\frac{B S}{2 \pi}\right)^{2} \cdot \zeta_{N-2}(s) .
$$

In this case there is a single recursive series. We define $\zeta_{N=0}(s)=0$, while our previous calculations have shown $\zeta_{N=1}(s)=\frac{B S}{\pi} \cdot\left(2 B / \mu^{2}\right)^{-s} \cdot \zeta_{R}(s)$. So we deduce $\zeta_{N=2}(s)=\left(\frac{B S}{\pi}\right)^{2} \cdot\left(2 B / \mu^{2}\right)^{-s} \cdot \zeta_{R}(s-1)$, which verifies our previous fourdimensional calculation. 
Without resorting to numerical computation, we can make some general observations regarding the $d$-dimensional case. Note that all the $d$-dimensional zeta functions are of the form

$$
\zeta_{d=2 N}(s)=B^{d / 2} \cdot\left(\frac{B}{\mu^{2}}\right)^{-s} \cdot \Omega \cdot f(s)
$$

in which case

$$
\mathcal{L}_{\text {eff }} \propto B^{d / 2}\left\{\ln \left(B / \mu^{2}\right) \cdot f(0)-f^{\prime}(0)\right\}
$$

so that the "folk theorem" generically holds for this class of fields. The "folk theorem" can fail if $f(0)$ happens to be zero; this is in fact exactly what happens for massless scalar particles in two dimensions. We remind the reader that the integrals (85) and (86) defining $f(s)$ make sense only for $\operatorname{Re}(s)>d / 2$. The value of $f(0)$ is defined by analytic continuation in $s$.

We also wish to point out that our recursive formulas (87) and (88) are not the only way of proceeding. The spectra are in this case sufficiently simple that explicit calculation in terms of Hurwitz zeta functions is possible.

\section{Odd Dimensionality}

In $d=2 N+1$ dimensions, we write the volume of spacetime as $\Omega_{d}=L \cdot \Omega_{2 N}$. The heat kernel is related to that in $2 N$ dimensions by $K_{d}(t)=\frac{L}{\sqrt{4 \pi t}} \cdot K_{2 N}(t)$, so that (paralleling the discussion of the three-dimensional case) one deduces

$$
\zeta_{d}(s)=\frac{L}{\sqrt{4 \pi}} \cdot \frac{\Gamma\left(s-\frac{1}{2}\right)}{\Gamma(s)} \cdot \zeta_{2 N}\left(s-\frac{1}{2}\right) .
$$

Consequently $\zeta_{d}^{\prime}(0)=-L \cdot \zeta_{2 N}\left(-\frac{1}{2}\right)$, and in the notation of equations (89) and (90) one has

$$
\mathcal{L}_{\text {eff }} \propto B^{d / 2} \cdot f\left(-\frac{1}{2}\right) .
$$

Though this calculation is carried out for the special case $\left|B_{1}\right|=\cdots=\left|B_{i}\right|=\cdots=$ $\left|B_{N}\right|=B$, we expect that for general fields the result $\mathcal{L}_{\text {eff }} \sim B^{d / 2}$ will remain true in any odd number of dimensions.

\section{Conclusions}

We have studied the effect of constant field strengths on the determinants of the Dirac operator and gauged Laplacian. We began with the topologically interesting cases of harmonic gauge potentials, before proceeding to monopole potentials with constant field strengths. In both these cases we obtained the spectrum of the squared Dirac operator and gauged Laplacian explicitly and calculated the corresponding zeta function directly. Computation of the effective action then reduces to a relatively simple application of special functions. 


\section{Appendix A. Hurwitz zeta Functions.}

To make this paper as self contained as possible, we shall present here a number of definitions and useful results related to zeta-function theory. Most of these results may be obtained by consulting Gradshteyn and Ryzhik. $\frac{32}{2}$ Other useful references include Weil 19 , Abramowitz and Stegun, 33 The Encyclopedia of Mathematics, 34 and the Bateman Manuscript Project. 35

The Hurwitz zeta function is defined by:

$$
\zeta_{H}(s ; x)=\sum_{n=0}^{\infty}(n+x)^{-s} .
$$

This series converges absolutely for $\operatorname{Re}(s)>1$, and the function so defined may be analytically continued to the entire complex plane. There is a single simple pole at $s=1$ and in that neighborhood $\zeta_{H}(s, x)=\frac{1}{s-1}-\psi(x)+o(s-1)$. Setting $x=1$ reproduces the ordinary Riemann zeta function,

$$
\zeta_{R}(s)=\zeta_{H}(s ; 1)=\sum_{n=1}^{\infty} n^{-s} .
$$

The Riemann eta function is closely related to the Riemann zeta function. It is defined by

$$
\eta(s)=\sum_{n=1}^{\infty}(-1)^{n+1} n^{-s}
$$

The Riemann eta function, being defined by an alternating series, is numerically much better behaved than the Riemann zeta function, and furthermore converges over a larger region of the complex $s$-plane $[\operatorname{Re}(s)>0]$. In terms of the eta function

$$
\zeta_{R}(s)=\frac{1}{1-2^{1-s}} \cdot \eta(s) .
$$

The Riemann zeta function satisfies the classical reflection formula

$$
\zeta_{R}(s)=\frac{(2 \pi)^{s}}{\pi} \cdot \sin \left(\frac{\pi s}{2}\right) \cdot \Gamma(1-s) \cdot \zeta_{R}(1-s) .
$$

By combining the reflection formula with an improved series representation for the zeta function given in terms of the eta function one may numerically compute Riemann's zeta function over the entire complex plane.

The Hurwitz zeta function may be evaluated in terms of Riemann's zeta function by using the binomial series to write

$$
\begin{aligned}
\zeta_{H}(s ; 1+x) & =\sum_{n=1}^{\infty} \sum_{l=0}^{\infty}\left(\begin{array}{c}
-s \\
l
\end{array}\right) \cdot n^{-s-l} \cdot x^{l} \\
& =\zeta_{R}(s)+\sum_{l=1}^{\infty}(-)^{l} \cdot \frac{s(s+1) \cdots(s+l-1)}{l !} \cdot \zeta_{R}(s+l) \cdot x^{l} .
\end{aligned}
$$


This series is convergent for $|x|<1$. An asymptotic expansion for large $x$ is provided by reference 35 [equation $1.18(9)]$ :

$$
\begin{aligned}
\zeta_{H}(s ; 1+x)= & \frac{1}{\Gamma(s)}\left[x^{1-s} \Gamma(s-1)+\frac{1}{2} x^{-s} \Gamma(s)\right. \\
& \left.+\sum_{k=1}^{n} \mathbf{B}_{\mathbf{2 k}} \frac{\Gamma(s+2 k+1)}{(2 k) !} x^{1-2-2 k}+O\left(x^{-1-s-2 n}\right)\right] .
\end{aligned}
$$

At special values of $s$ more information is available. The value of the Hurwitz zeta function at non-positive integers is known in terms of the Bernoulli polynomials

$$
\zeta_{H}(-n ; x)=-\frac{\mathbf{B}_{\mathbf{n}+\mathbf{1}}(x)}{n+1}=-\frac{\mathbf{B}_{\mathbf{n}+\mathbf{2}}^{\prime}(x)}{(n+1)(n+2)},
$$

in particular,

$$
\begin{gathered}
\zeta_{H}(0 ; x)=\frac{1}{2}-x ; \\
\zeta_{H}(-1 ; x)=-\frac{1}{2} x^{2}+\frac{1}{2} x-\frac{1}{12}=-\frac{1}{2}\left(x-\frac{1}{2}\right)^{2}+\frac{1}{24} .
\end{gathered}
$$

The derivative of the Hurwitz zeta function at $s=0$ is known to be

$$
\zeta_{H}^{\prime}(0 ; x)=\ln (\Gamma(x) / \sqrt{2 \pi}) .
$$

Derivatives at other values of $s$ are not given in the standard tables. One may make some progress by noting that

$$
\frac{\partial \zeta_{H}(s ; 1+x)}{\partial x}=-s \cdot \zeta_{H}(s+1 ; 1+x)
$$

so that

$$
\frac{\partial \zeta_{H}^{\prime}(s ; 1+x)}{\partial x}=-\zeta_{H}(s+1 ; 1+x)-s \cdot \zeta_{H}^{\prime}(s+1 ; 1+x) .
$$

This recursion relation, when applied to the case $s=-1$ yields

$$
\begin{gathered}
\zeta_{H}^{\prime}(-1 ; 1+x)=\zeta_{R}^{\prime}(-1)+\frac{1}{2}\left(x^{2}+x\right)+\int_{0}^{x} \ln (\Gamma(1+y) / \sqrt{2 \pi}) d y \\
=\zeta_{R}^{\prime}(-1)+\frac{1}{2}\left(x^{2}+x\right)-\frac{1}{2} x \ln (2 \pi)+x \ln \Gamma(1+x) \\
-\int_{0}^{x} y \psi(1+y) d y
\end{gathered}
$$

This is the best analytic result that we have been able to obtain. For $|x|<1$ we may make the convergent expansion

$$
\begin{gathered}
\zeta_{H}^{\prime}(-1 ; 1+x)=\zeta_{R}^{\prime}(-1)+\frac{1}{2}\left(x^{2}+x\right)-\frac{1}{2} x \ln (2 \pi)+x \ln \Gamma(1+x) \\
+\frac{1}{2} \gamma x^{2}-\sum_{k=2}^{\infty}(-)^{k} \frac{\zeta_{R}(k) x^{k+1}}{(k+1)} .
\end{gathered}
$$


For $|x|$ large, a computationally useful asymptotic expansion may be found in expositions by Elizalde and Soto $30 \mid 31$ :

$$
\begin{aligned}
\zeta_{H}^{\prime}(-1 ; x)=\left(\frac{1}{2} x^{2}\right. & \left.-\frac{1}{2} x+\frac{1}{12}\right) \cdot \ln x-\frac{1}{4} x^{2}+\frac{1}{12} \\
& -\sum_{k=1}^{n-1} \frac{\mathbf{B}_{\mathbf{2}+\mathbf{2}}}{(2 k+2)(2 k+1)(2 k)} \cdot x^{-2 k}+O\left(x^{-2 n}\right) .
\end{aligned}
$$

(But note theat the final sign is erroneously displayed in reference 30 .) We have resorted to numerical methods to evaluate

$$
\begin{array}{ll}
\zeta_{R}\left(\frac{3}{2}\right) \approx 2.612375 ; & \zeta_{R}\left(-\frac{1}{2}\right) \approx-0.207886 ; \\
\zeta_{R}^{\prime}(2) \approx-0.937548 ; & \zeta_{R}^{\prime}(-1) \approx-0.165421 .
\end{array}
$$

Finally, we note that $\Gamma\left(\frac{1}{2}\right)=\sqrt{\pi}$, and that Stirling's approximation is

$\ln (\Gamma(x) / \sqrt{2 \pi})=\left(x-\frac{1}{2}\right) \ln (x)-x+\frac{1}{12 x}+\sum_{k=1}^{n-1} \frac{\mathbf{B}_{\mathbf{2 k}+\mathbf{2}}}{(2 k+2)(2 k+1)} x^{-1-2 k}+O\left(x^{-2 n}\right)$.

It is often sufficient to use the simpler form

$$
\ln (\Gamma(x) / \sqrt{2 \pi})=\left(x-\frac{1}{2}\right) \ln (x-1)-(x-1)+O(1 / x) .
$$

\section{References}

1. H. Euler and W. Heisenberg, Z. Physik 98 (1936) 714.

2. V. Weisskopf, Kgl. Danske Videnskab. Selskabs. Mat.-fys. Medd. 14 No.6 (1936).

3. J. Schwinger, Phys. Rev. 82 (1951) 664.

4. L. Alvarez-Gaume, G. Moore, and C. Vafa, Commun. Math. Phys. 106 (1986) 1.

5. L. Alvarez-Gaume, J.-B. Bost, G. Moore, P. Nelson, and C. Vafa, Commun. Math. Phys. 112 (1987) 503.

6. E. D'Hoker, and D.H. Phong, Nuc. Phys. B278 (1986) 226.

7. E. D'Hoker, and D.H. Phong, Comm. Math. Phys. 104 (1986) 537.

8. J. Schwinger, Phys. Rev. 128 (1962) 2425.

9. S. Blau, M. Visser, and A. Wipf, Int. J. Mod. Phys. A4 (1989) 1467.

10. N.D. Birrel and P.C. Davies, Quantum Fields in Curved Space, (Cambridge University Press, Cambridge, 1982).

11. S. Blau, M. Visser, and A. Wipf, Phys. Lett. B209 (1988) 209.

12. L.S. Brown and D.B.Creamer, Phys. Rev. D18 (1978) 3695.

13. E. Corrigan, P. Goddard, H. Osborn, and S. Templeton, Nucl. Phys. B159 (1979) 469.

14. H. Osborn, Nucl. Phys. B159 (1979) 497.

15. W. Dittrich and M. Reuter, Effective Lagrangians in Quantum Electrodynamics, (Springer-Verlag, Berlin, 1984), Vol 220 of Lecture Notes in Physics.

16. S. K. Blau, M. Visser, and A. Wipf, Nuclear Physics B310 (1988) 163.

17. P. Epstein, Math. Ann. 56 (1903) 516.

18. P. Epstein, Math. Ann. 63 (1907) 205.

19. A. Weil, Elliptic Functions according to Eisenstein and Kronecker, (Springer-Verlag, Berlin, 1976).

20. L. Euler, Werke, Ser. I, Vol. VII, Ch XV, §274.

21. L. Kronecker, Werke, Vol IV, p. 222, (1863). 
22. F. Oberhettinger, Fourier Expansions, (Academic, New York, 1973).

23. R. Balian, C.Itzykson, and J.B.Zuber, Phys. Rev. D17 (1978) 1041.

24. L. Kronecker, Berliner Sitzungsberichte, p. 53, (1889).

25. D. Mumford, Tata Lectures on Theta, (Birkhäuser, Boston, 1983).

26. E. Jahnke and F. Emde, Tables of functions, 4th edition, (Dover, New York, 1956).

27. A. N. Redlich, Phys. Rev. Lett. 52 (1984) 18.

28. A. J. Niemi and G. Semenoff, Phys. Rev. Lett. 51 (1982) 2077.

29. A. N. Redlich, Phys. Rev. D29 (1984) 2366.

30. E. Elizalde, Nucl. Phys. B243 (1984) 398.

31. E. Elizalde and J. Soto, Ann. Phys. (N.Y.) 162 (1985) 192.

32. I.S. Gradshteyn, and I.M. Ryzhik, Table of Integrals, Series, and Products, (Academic, New York, 1980).

33. M. Abramowitz, and I.A. Stegun, Handbook of Mathematical Functions, (Dover, New York, 1970).

34. Encyclopedic Dictionary of Mathematics, edited by S. Iyanaga and Y. Kawada, (MIT Press, Cambridge, 1977).

35. The Bateman Manuscript Project, Higher Transcendental Functions, edited by A. Erdélyi, W. Magnus, F. Oberhettinger, and F.G. Tricomi, (McGraw-Hill, New York, 1953-55), Vol 3. 